\title{
Mitchell Osteotomy for Hallux Valgus Using Trans-Osseous Capsulorrhaphy for Stabilization
}

\author{
Adnan A Faraj FRCS (Orth)* \\ Associate Professor, Orthopaedic Department, Azadi Hospital, Kirkuk Medical School, Kirkuk, Airedale \\ Foundation Trust, Keighley, UK
}

*Corresponding author: Adnan A Faraj, Associate Professor, Orthopaedic Department, Azadi Hospital, Kirkuk Medical School, Kirkuk, Iraq Surgeon, Airedale Foundation Trust, Keighley, UK, Tel: 004-474-382-29-521

\begin{abstract}
Background: The fixation method of Mitchell's osteotomy after displacement is controversial. In the current study, we evaluated a previously described technique of using transosseous capsulorrhaphy. In this series, foot plaster slipper was used for six weeks.

Material and methods: Twenty-seven feet (21 patients) underwent the technique for symptomatic hallux valgus was included in this study. Retrospective and consecutive series. Mitchell's step cut osteotomy was performed without metal fixation, trans osseous capsulorrhaphy was used for fixation.

Results: At 15 months follow-up, the results were satisfactory in 23 feet, fair in three patients and poor in one patient. Mean HVA correction was $17^{\circ}$ and IMA correction of $5.4^{\circ}$ this is considered to be significant.

Conclusion: The main advantage of the described technique is that it can be used in a less sophisticated surgical unit, without the need for internal fixation implants and image intensifier.
\end{abstract}

\section{Keywords}

Hallux valgus, Mitchell's, Osteotomy, Trans-osseous, Capsulorrhaphy

\section{Introduction}

More than 300 surgical procedures have been described over the years for the correction of HV deformity, often a metallic screw fixation is used to stabilize osteotomy. Metallic screw fixation is now the most frequently used fixation method for modified chevron osteotomies. However, elective implant removal due to irritation of metallic screws has been reported in up to $26.9 \%$ of patients in reported series [1]. A second operation decreases patient satisfaction and increases the overall cost of the treatment. Metal insertion remains to have concerns. Most of the historical osteotomy were described without metal fixation and had good outcome, some popular types of osteotomy are Mitchell, Chevron and other osteotomies. The Mitchell operation for hallux valgus was first described in the literature in 1945 by Hawkins and associates [2]. Mygind, in 1952, described a similar procedure [3].

Mitchell subsequently published an article in 1958 describing this procedure, and from this point on it became known as the Mitchell bunionectomy. Mitchell's original description of this procedure included an osteotomy of the distal portion of the first metatarsal, lateral displacement and angulation of the head of the metatarsal, and exostectomy and capsulorrhaphy [3].

The method of fixation, as described by Mitchell and others utilizing suture material, although reported to have adequate results seems to lend itself to potential complications. The most serious complication is dorsal displacement of the capital fragment after fixation is in place. This may lead to lesser metatarsalgia, lesser metatarsal lesions, and hallux limitus or rigidus. With the advances in fixation devices available to the surgeon over the years since the Mitchell procedure was first described, better and sounder fixation principles are available that will yield better results. The techniques used are cross-Kirshner wire fixation, biodegradable

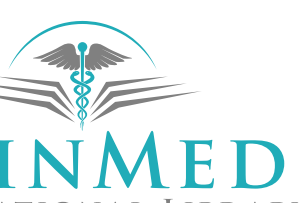

INTERNATIONAL LIBRARY

Citation: Faraj AA (2019) Mitchell Osteotomy for Hallux Valgus Using Trans-Osseous Capsulorrhaphy for Stabilization. Int J Foot Ankle 3:026.

Accepted: March 18, 2019; Published: March 20, 2019

Copyright: (C) 2019 Faraj AA. This is an open-access article distributed under the terms of the Creative Commons Attribution License, which permits unrestricted use, distribution, and reproduction in any medium, provided the original author and source are credited. 
wire fixation, Herbert screw, A-O screws and other techniques [4-9]. The advocates of internal fixation claim that the stability of the Mitchell procedure is compromised when relying on the lateral spicule, soft tissue, and splinting techniques to maintain correction. Internal fixation, however, can be associated with complications; the fixation is not always secure, the technique is difficult, and implant can damage the articular surface or cause pain if the end is prominent and may require another operation to remove. This is why finding alternative reliable techniques to internal fixation to secure the osteotomy, like the technique pursued in the current study, becomes attractive.

In the current retrospective study, we report the clinical and radiological outcome of Mitchell's osteotomy using trans-osseous capsulorrhaphy for fixation without internal fixation. Technique has been previously described [10].

\section{Material and Methods}

Twenty-seven feet (21 patients) who underwent modified Mitchell's osteotomy for symptomatic hallux valgus were included between 2009 and 2014 consecutively. There were 19 female, and 2 male patients. The mean age of 47 -years (17-52 years). One patient lost follow up and was excluded from this series. The left foot was operated on in 16 and the right in 11. Staged bilateral procedures were performed in 6 patients (total 27 feet). The indication for surgery was bunion pain, and pressure symptoms affecting the second toe because of an under or overlying hallux which has failed to respond to shoe modifications and silicon spacers in between the hallux and the $2^{\text {nd }}$ toe.

Standing plain X-rays (anteroposterior and lateral) was performed in each patient, looking for the hallux valgus angle (HVA), intermetatarsal angle (IMA), the length of the first metatarsal and for excluding osteoarthritic changes of the metatarsophalangeal joint (MTPJ).

Patients with gout, elderly with what seems on plain $\mathrm{X}$-ray osteopenia suggesting poor bone quality and rheumatoid patients were excluded.

In our series, we also excluded patients with lateral forefoot pain or dorsolateral big toe metatarsophalangeal joint (MTPJ) pain from the series. $5 \mathrm{~mm}$ or more of first metatarsal Shortening compared to the second metatarsal were also excluded from the study. Intermetatarsal angle (IMA) of more than $25^{\circ}$ and metatarsophalangeal angle more than $44^{\circ}$ were also excluded. The modification is described below (10) (Figure 1 and Figure 2).

\section{Technique}

It is our practice to perform the operation under general anaesthetics (unless the patient is unfit) and as a day case. A pneumatic above knee tourniquet is

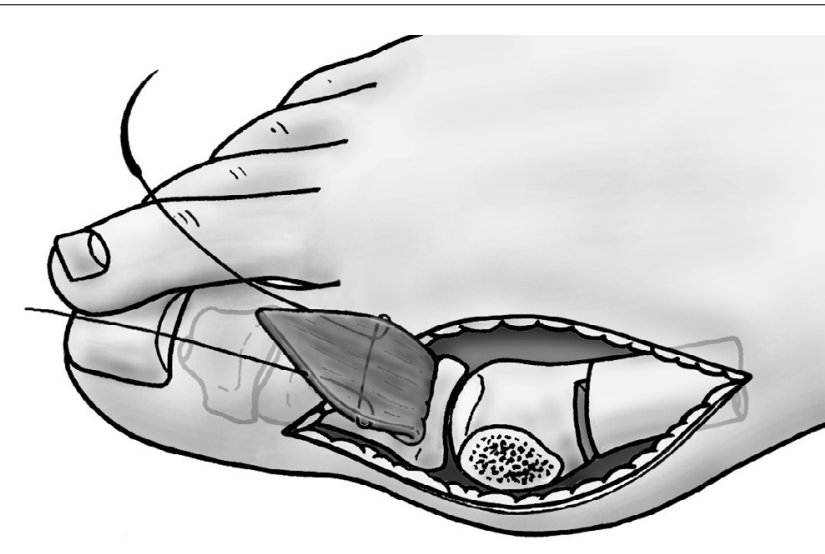

Figure 1: Graphic demonstration of the technique showing the capsular flap and the step cut osteotomy.

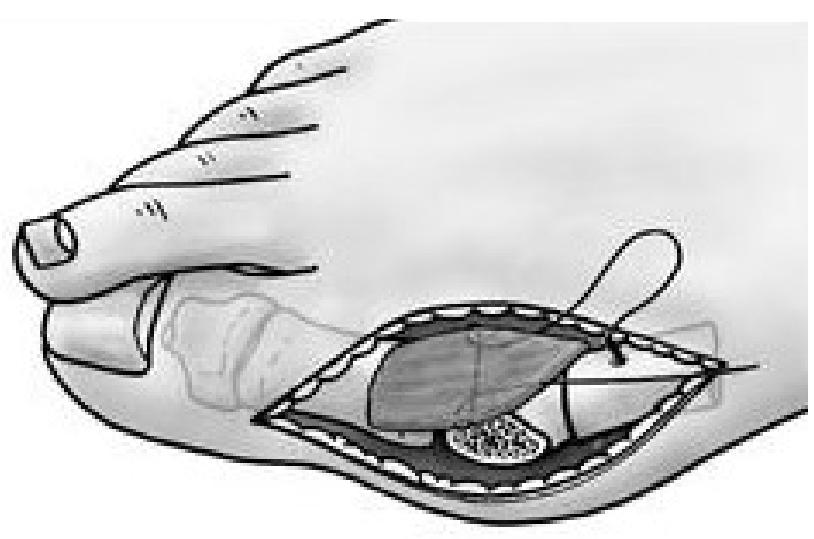

Figure 2: Trans-osseous capsulorrhaphy: Kessler Number 1-0 Vicryl is passed through the $\mathrm{V}=$ shaped capsular cut and passed through a drill hole in the proximal part of the osteotomy to tighten up after the displacement of the osteotomy.

used and a single dose of prophylactic antibiotics (cephalosporin) is used. Through a medial midline incision. The dorsal digital nerve is identified and protected. A V-shaped MTPJ capsular flap is created with the base of the triangle attached to the proximal phalanx. The tip of the triangle is raised from the first metatarsal. A Kessler type suture of 1-0 Vicryl is passed through the capsule from the tip of the triangle to use as a stay suture (the needle is not removed). The metatarsophalangeal joint capsule is pulled away with this stay suture to aid in the exposure of the joint. Step-cut Mitchell's osteotomy is carried out as usual (Figure 1). A $2 \mathrm{~mm}$ drill is passed through the first metatarsal just proximal to the osteotomy in a vertical fashion and on the medial side. The needle of the stay suture of the capsule is passed through this hole after displacing the osteotomy and the Vicryl suture is securely knotted to stabilise the fixation (Figure 2). When the deformity was severe and the correction inadequate despite lateral displacement, a lateral release was carried out; this was necessary in 3 patients. No intraoperative X-ray is used, the correction of the deformity was assessed mainly on appearance and the percentage of displacement (Figure 3). 

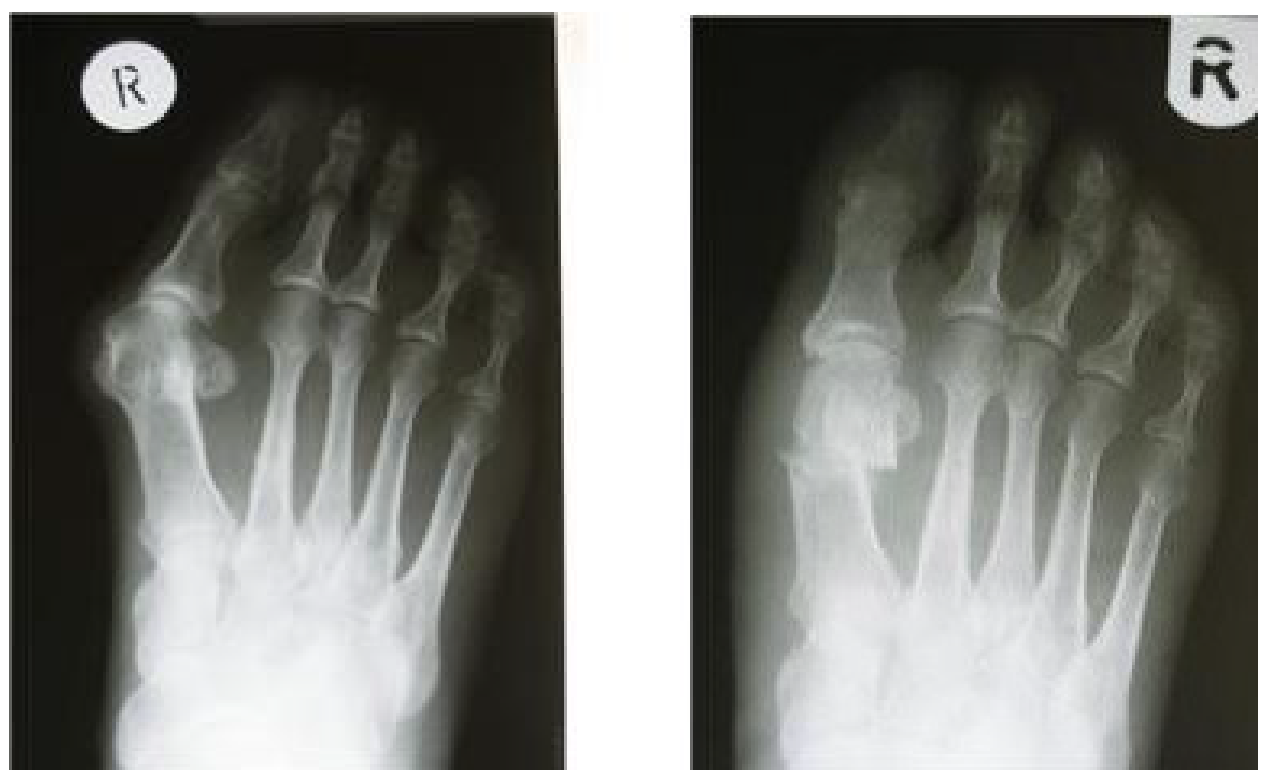

Figure 3: Pre and post Anter-posterior plain X-ray of the foot six months following Mitchell's osteotomy.

The wound is closed using 3-0 Vicryl. Local long acting anaesthetic ankle block or local infiltration is used at the end of the operation for pain relief.

The patient is allowed mobilization and weight bearing on the foot avoiding the forefoot. Chemical thromboprophylaxis was used in patients with high risk in the form of subcutaneous Fragmin for 4 weeks. A slipper light cast was applied (involving the hallux and the heel). The cast was removed 6 weeks after surgery, or when necessary (tight or concerned about the wound). Radiographs of the foot (anteroposterior and lateral is performed to check for correction of the deformity, and healing of the osteotomy.

Check AP and lateral $\mathrm{X}$-rays is repeated if there are concerns about healing or the osteotomy or when any complication arise at 3-, 6- and 12-months following surgery was performed.

\section{Results}

Mean follow-up was 15 months (9-24 months). The assessment involved medical notes and $\mathrm{X}$-ray review. The assessment therefore was clinical and radiological. The American foot and ankle society scoring system was used. Preoperative pain was arising from the bunions. The mean improvement in pain was 33 Score (29-40).

The clinical assessment included questioning about pain, shoe wear prior and after surgery. These patients did have problems with even comfortable shoes prior to surgery. Shoe wear improvement after surgery was significant; the patients were however advised to wear high toe box shoes and silicon spacer to avoid recurrence of deformity because of tight shoes.

All but one patient was very satisfied with the shoe wear and the shape of the foot. Six of our patients had bilateral hallux valgus, they came back to have the other side done as they were pleased with the outcome of the first surgery.

The range of movement of the metatarsophalangeal joint of the hallux prior and after surgery was assessed using goniometry, in all apart from 3 patients; the range of movement was satisfactory, often after cast removal all these patients were stiff at the MTPJ, but within a mean period of 3 weeks after cast removal (2-17 weeks) the range of movement improved. The results were considered satisfactory, when the pain was better, the shape of the toe was considered to be good by the patient and the range of movement of the big toe MTPJ was as good as $80 \%$ of the range of movement prior to surgery. Satisfactory radiological outcome was when the HVA correction was good (more than 15 degrees), the osteotomy was healing with no significant shortening of the first metatarsal (6 mm shortening was the cut-off) and no dorsal displacement of the first metatarsal head. Fair results were patients with good clinical and radiological correction of the deformity apart from the range of movement (had stiffer MTPJ movement compared to preoperative range of movement). Poor results were for patients with complications, metatarsalgia, recurrence, infection, neurological deficit, wound dehiscence, and significant radiological shortening of the first metatarsal (more than $6 \mathrm{~mm}$ with dorsal displacement of the head of the first metatarsal).

The results were satisfactory in 23 patients, fair in three patients who mainly complained of stiffness of the big toe MTPJ movement; the metatarsophalangeal joint of the hallux became stiffer than before surgery mean dorsiflexion of $32^{\circ}\left(30^{\circ}-45^{\circ}\right)$.

The result was poor in one patient who developed metatarsalgia, this patient had $6 \mathrm{~mm}$ shortening of the first metatarsal and partially lost the reduction, however the HVA correction remained better than before surgery $\left(43^{\circ}\right.$ to $\left.23^{\circ}\right)$. No further treatment was required. 
Radiological assessment included the comparison of pre and post late standing $\mathrm{X}$-ray radiological measurement of HVA, IMA, Union of the fracture and any other abnormality. The mean period to union of the osteotomy (bone healing across the osteotomy with trabecular bone formation) was 8 weeks (6-12 weeks).

\section{Results and statistics}

$\mathrm{Z}$ score calculator to $\mathrm{P}$ value conversion was used in the analysis of the measurements. Mean HV angle before surgery was $31.3^{\circ}\left(\mathrm{sd}=6.1\right.$; range $=25^{\circ}-43^{\circ}$ ) compared with $12.4^{\circ}\left(\mathrm{sd}=4.5\right.$; Range $=5^{\circ}-22^{\circ}$ ) after the osteotomy; this was significant $Z=4.54(P<0.01)$.

Mean IM angle before surgery was $14.9^{\circ}(\mathrm{sd}=3.7$; range $\left.=10^{\circ}-24^{\circ}\right)$ and $\mathrm{IMA}$ became $10.1^{\circ}(\mathrm{sd}=2.3$; range $\left.=5^{\circ}-20^{\circ}\right)$; this was significant $Z=4.55(P<0.01)$.

The mean shortening of the first metatarsal after Mitchell's osteotomy was $4 \mathrm{~mm}(2-7 \mathrm{~mm}$ ) (Figure 3 and Table 1).

The mean dorsiflexion of metarasal head on lateral view after osteotomy was $1.3^{\circ}\left(0-5^{\circ}\right)$. Those cases with relatively higher shortening of the first metatarsal in the series had relatively higher incidence of dorsiflexion angle.

\section{Complications}

One patient developed metatarsalgia; this patient had $6 \mathrm{~mm}$ shortening of the first metatarsal and partially lost the reduction, however the HVA correction remained better than before surgery $\left(43^{\circ}\right.$ to $\left.23^{\circ}\right)$. No further treatment was required. Another patient developed stitch abscess causing redness and swelling for 3 weeks, short term oral antibiotics was prescribed, and the symptoms resolved completed. Undissolved subcuticular Vicryl suture worked their way out in 2 patients. In one patient the stitch had to be removed through a small skin incision under local anaesthetics. In three patients the metatarsophalangeal joint of the hallux became stiffer than before surgery. Mean dorsiflexion Satisfactory range of dorsiflexion of the MTPJ was considered to be of a mean $40^{\circ}\left(35-50^{\circ}\right)$, and a mean plantar flexion in these patients were $20^{\circ}$ (Table 2 ).

Table 1: Radiological assessment following surgery assessing.

\begin{tabular}{|c|c|c|c|c|c|c|}
\hline & Pre-HVA $^{\circ}$ & Post-HVA ${ }^{\circ}$ & Pre-IMA' & Post-IMA ${ }^{\circ}$ & $\begin{array}{l}\text { Shortening of first } \\
\text { metatarsal (mm) }\end{array}$ & $\begin{array}{l}\text { The angle between First metatarsal } \\
\text { head and shaft (lat view) }\end{array}$ \\
\hline 1 & $35^{\circ}$ & $5^{\circ}$ & $15^{\circ}$ & $11^{\circ}$ & 5 & 0 \\
\hline 2 & $24^{\circ}$ & $14^{\circ}$ & $13^{\circ}$ & $10^{\circ}$ & 4 & 0 \\
\hline 3 & $30^{\circ}$ & $10^{\circ}$ & $16^{\circ}$ & $13^{\circ}$ & 4 & 1 \\
\hline 4 & $30^{\circ}$ & $8^{\circ}$ & $14^{\circ}$ & $10^{\circ}$ & 5 & 2 \\
\hline 5 & $24^{\circ}$ & $10^{\circ}$ & $10^{\circ}$ & $9^{\circ}$ & 2 & 0 \\
\hline 6 & $25^{\circ}$ & $8^{\circ}$ & $13^{\circ}$ & $10^{\circ}$ & 3 & 0 \\
\hline 7 & $32^{\circ}$ & $16^{\circ}$ & $14^{\circ}$ & $10^{\circ}$ & 3 & 0 \\
\hline 8 & $30^{\circ}$ & $12^{\circ}$ & $13^{\circ}$ & $8^{\circ}$ & 4 & 2 \\
\hline 9 & $30^{\circ}$ & $10^{\circ}$ & $10^{\circ}$ & $7^{\circ}$ & 5 & 2 \\
\hline 10 & $28^{\circ}$ & $5^{\circ}$ & $14^{\circ}$ & $8^{\circ}$ & 6 & 5 \\
\hline 11 & $22^{\circ}$ & $8^{\circ}$ & $11^{\circ}$ & $10^{\circ}$ & 2 & 0 \\
\hline 12 & $35^{\circ}$ & $10^{\circ}$ & $14^{\circ}$ & $8^{\circ}$ & 6 & 2 \\
\hline 13 & $22^{\circ}$ & $10^{\circ}$ & $15^{\circ}$ & $8^{\circ}$ & 5 & 2 \\
\hline 14 & $30^{\circ}$ & $11^{\circ}$ & $15^{\circ}$ & $9^{\circ}$ & 6 & 3 \\
\hline 15 & $32^{\circ}$ & $16^{\circ}$ & $12^{\circ}$ & $9^{\circ}$ & 5 & 2 \\
\hline 16 & $42^{\circ}$ & $16^{\circ}$ & $14^{\circ}$ & $10^{\circ}$ & 5 & 2 \\
\hline 17 & $30^{\circ}$ & $14^{\circ}$ & $12^{\circ}$ & $10^{\circ}$ & 4 & 0 \\
\hline 18 & $43^{\circ}$ & $18^{\circ}$ & $14^{\circ}$ & $11^{\circ}$ & 6 & 3 \\
\hline 19 & $32^{\circ}$ & $17^{\circ}$ & $18^{\circ}$ & $10^{\circ}$ & 5 & 2 \\
\hline 20 & $42^{\circ}$ & $18^{\circ}$ & $18^{\circ}$ & $12^{\circ}$ & 3 & 0 \\
\hline 21 & $35^{\circ}$ & $12^{\circ}$ & $16^{\circ}$ & $14^{\circ}$ & 4 & 0 \\
\hline 22 & $43^{\circ}$ & $23^{\circ}$ & $16^{\circ}$ & $10^{\circ}$ & 6 & 3 \\
\hline 23 & $30^{\circ}$ & $15^{\circ}$ & $20^{\circ}$ & $12^{\circ}$ & 7 & 5 \\
\hline 24 & $35^{\circ}$ & $16^{\circ}$ & $26^{\circ}$ & $18^{\circ}$ & 2 & 0 \\
\hline 25 & $32^{\circ}$ & $17^{\circ}$ & $24^{\circ}$ & $14^{\circ}$ & 3 & 0 \\
\hline 26 & $24^{\circ}$ & $8^{\circ}$ & $12^{\circ}$ & $10^{\circ}$ & 3 & 0 \\
\hline 27 & $28^{\circ}$ & $8^{\circ}$ & $15^{\circ}$ & $10^{\circ}$ & 3 & 0 \\
\hline
\end{tabular}


Table 2: Pre and postoperative range of movement of the metatarsophalangeal joint of the hallux. In three patients the metatarsophalangeal joint of the hallux became stiffer than before surgery mean dorsiflexion and plantar flexion.

\begin{tabular}{|l|l|l|l|l|}
\hline $\begin{array}{l}\text { Number of } \\
\text { feet }\end{array}$ & $\begin{array}{l}\text { Preoperative range of } \\
\text { dorsiflexion }\end{array}$ & $\begin{array}{l}\text { Postoperative range of } \\
\text { dorsiflexion }\end{array}$ & $\begin{array}{l}\text { Preoperative range of } \\
\text { plantar flexion }\end{array}$ & $\begin{array}{l}\text { Postoperative range of } \\
\text { plantar flexion }\end{array}$ \\
\hline 24 & Mean $42^{\circ}\left(40-45^{\circ}\right)$ & Mean $45^{\circ}\left(40-50^{\circ}\right)$ & Mean $32^{\circ}\left(30-40^{\circ}\right)$ & Mean $25^{\circ}\left(15-40^{\circ}\right)$ \\
\hline 3 & Mean $40^{\circ}\left(35-50^{\circ}\right)$ & $42^{\circ}\left(30-45^{\circ}\right)$ & Mean $30^{\circ}\left(25-35^{\circ}\right)$ & Mean $20^{\circ}\left(10-350^{\circ}\right)$ \\
\hline
\end{tabular}

\section{Discussion}

Although the metatarsal head is often intrinsically stable with step-cut Mitchell's osteotomy, secure existence of new techniques, Mitchell's osteotomy in the treatment of Hallux valgus remains to be used in our era and indications has expanded from mild to moderate hallux valgus surgery, to moderate and severe hallux valgus surgery $[1,9]$ this partly is because of the simplicity of the technique and relative stability of the osteotomy regardless of whether internal fixation or suture fixation, is used.

Internal fixation is a routine to prevent displacement of the osteotomy. Despite the current trend using implants to fix Mitchell's osteotomy, there has been concurrent concerns and complications related to the internal fixation. Some of the complications are related to the type of osteotomy rather and not necessarily the methods of fixation of the osteotomy. The disadvantage of this, however, can undermine the procedure. Passing a screw across the osteotomy is daunting. It is almost impossible to pass a screw perpendicular to the plane of osteotomy to achieve compression [11]. Bonner described using a jig to ensure accurate placement of a Herbert screw for fixation of Mitchell's osteotomy [6]. This suggestion is interesting since it may also eliminate skin irritation caused by the prominent head of an $A O$ screw [6]. The screw can also penetrate the metatarsal head, hence fluoroscopic image seems necessary. The latter exposes patient to radiation. Where the screw inserted at the medial metatarsal neck, there is potential stress area with possible stress fracture. Even if all goes well, sometimes the screw head is prominent causing pressure inside the shoes; this may necessitate its removal and further surgery. Herbert screw is used to eliminate the possibility of prominent screw head. Future reconstruction of the big toe when required is also difficult in the presence of implants. Often screws have to be removed when the osteotomy is stabilized using screw fixation [4], and in another series of 161 feet, were pins were used for fixation of Mitchell's osteotomy, there were 13 pin tract infections, two delayed unions, and two correction losses and metatarsalgia of the lesser toes, which occurred in 20 feet (12.4\%), leading to some dissatisfaction [12].

The concern using trans-osseous capsulorrhaphy is displacement of the osteotomy; this will result in loss of reduction and shortening of the first metatarsal. Although Mitchell found no correlation between the amount of shortening and second metatarsalgia, since his report other authors have noted this as being a problem; more than $7 \mathrm{~mm}$. of shortening appears to yield poor results [9]. The mean reduction in length of the first metatarsal is 4 to $5 \mathrm{~mm}$ in most of the series reporting the outcome of Mitchell's osteotomy [8]. The mean shortening of the first metatarsal after Mitchell's osteotomy in this series was similar to what is reported using implant fixation. The technique using trans-osseous capsulorrhaphy, described in this paper is reproducible, easy to do and secure. The capsule of the MTPJ is thick and strong, when pulled across the metatarsal through a drill hole, it offers a tension band effect preventing dorsal displacement of the head of the first metatarsal and maintaining the reduction without the need for implant fixation.

The correction of the hallux valgus in this series was satisfactory. The upper limits of the hallux abductus angle (HAA) in which a good result can be obtained have been consistent, being somewhere in the $30^{\circ} \pm 5^{\circ}$ range [11]. Similarly, angles greater than $40^{\circ}$ have been reported to be associated with poor results [9]. The average amount of correction of hallux abductus with the Mitchell's osteotomy is dependent on the surgeon performing the operation. Averages of correction have been stated in the literature, but they have appeared very sporadically [1-13].

The mean $\mathrm{HV}$ angle before surgery in our series was $31.3^{\circ}\left(25^{\circ}-43^{\circ}\right)$ and mean IM angle of $15^{\circ}\left(10^{\circ}-26^{\circ}\right)$. The mean post-operative $\mathrm{HV}$ angle became $14^{\circ}\left(5^{\circ}-22^{\circ}\right)$ and the mean intermetatarsal angle became $10^{\circ}\left(5^{\circ}-20^{\circ}\right)$.

In our series we avoided Mitchell's osteotomy in patients with lateral metatarsalgia -forefoot pain; as Mitchell's osteotomy shortens the first metatarsal and worsens metatarsalgia. Other osteotomies (Scarf osteotomy, basal) were used instead.

Despite the shortfalls of our paper for not being a randomised controlled study addressing all the variables, we feel, the results using trans-osseous capsulorrhaphy for fixation of Mitchell's osteotomy is effective and the results are not inferior to the series where implants have been used for fixation. It is a useful technique in the developing countries where expertise and finance can be an issue.

\section{Conflict of Interest}

None. 


\section{Financial Gains}

None.

\section{References}

1. Jentzsch $T$, Renner $N$, Niehaus $R$, Farei Campagna J, Deggeller M, et al. (2018) The influence of the number of screws and additional surgical procedures on outcome in hallux valgus treatment. J Orthop Surg Res 13.

2. Hawkins HB, Mitchell CL, Hedrick DW (1945) Correction of hallux valgus by metatarsal osteotomy. J Bone Joint Surg 27: 387-394.

3. Mygind H (1952) Operations for hallux valgus. J Bone Joint Surg 34: 529-536.

4. Shih Hao Huang, Yuh Min Cheng, Yuh Min Cheng, Huang PJ (2012) Modified mitchell osteotomy with screw fixation for correction of hallux valgus. Foot Ankle Int 33: 1098-1102.

5. Calder JDF, Hollingdale JP, Pearse MF (1999) Screw versus suture fixation of Mitchell's osteotomy: A prospective, randomised study. J Bone Joint Surg Br 81: 621-624.

6. Bonner AC Jr (1986) Rigid internal fixation of the mitchell- hawkins osteotomy/bunionectomy with the herbert bone screw. J Foot Surg 25: 390-393.

7. Meier PJ, Kenzora JE (1985) The risks and benefits of first metatarsal osteotomies. Foot Ankle 6: 7-17.

8. Broughton NS, Winson IG (1990) Keller's arthroplasty and mitchell osteotomy: A comparison with first metatarsal osteotomy of the long-term results for hallux valgus deformity in the female. Foot Ankle 10: 201-205.

9. Kalender AM, Uslu M, Bakan B, Ozkan F, Erturk C, et al. (2013) Mitchell's ostotomy with mini-plate and screw fixation for hallux valgus. Foot Ankle Int 34: 238-243.

10. Jehanzeb T, Dosani A, Mann CF, Faraj AA (2005) Clinical tip: Transosseous capsular stabilization of modified mitchell osteotomy. Foot Ankle Int 26: 664-666.

11. Faraj AA (1999) Screw versus suture fixation of mitchell's osteotomy. J Bone Joint Surg Br 81: 1084-1085.

12. CH Kuo, PJ Huang, YM Cheng, KY Huang, Chen TB, et al. (1998) Modified mitchell osteotomy for hallux valgus. Foot Ankle Int 19: 585-589.

13. Dennis NZ, Das De S (2011) Modified mitchell's osteotomy for moderate to severe hallux valgus--an outcome study. J Foot Ankle Surg 50: 50-54. 